\title{
Optical Demultiplexer Device Operating in the Visible Spectrum
}

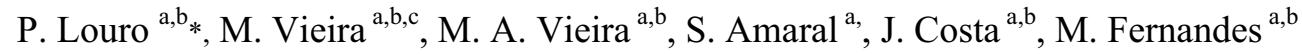 \\ ${ }^{a}$ Electronics Telecommunications and Computer Dept, ISEL, 1949-107, Lisbon, Portugal \\ ${ }^{b}$ CTS-UNINOVA, Quinta da Torre, Monte da Caparica, 2829-516, Caparica, Portugal \\ ${ }^{c}$ DEE-FCT-UNL, Quinta da Torre, Monte da Caparica, 2829-516, Caparica, Portugal
}

\begin{abstract}
In this paper we present results on the use of multilayered a-SiC:H heterostructures as a device for wavelength-division demultiplexing of optical signals. These devices are useful in optical communications applications that use the wavelength division multiplexing technique to encode multiple signals into the same transmission medium. The device is composed of two stacked p-i-n photodiodes, both optimized for the selective collection of photo generated carriers. Band gap engineering was used to adjust the photogeneration and recombination rates profiles of the intrinsic absorber regions of each photodiode to short and long wavelength absorption and carrier collection in the visible spectrum. The photocurrent signal using different input optical channels was analyzed at reverse and forward bias and under steady state illumination. A demux algorithm based on the voltage controlled sensitivity of the device was proposed and tested. An electrical model of the WDM device is presented and supported by the solution of the respective circuit equations. Other possible applications of the device in optical communication systems are also proposed.
\end{abstract}

(C) 2010 Published by Elsevier Ltd. Open access under CC BY-NC-ND license.

Keywords: optical sensors; wavelength division multiplexing-demultiplexing ; electrical simulation; optical communications.

\section{Introduction}

Wavelength division multiplexing (WDM) devices are used when different optical signals are encoded in the same optical transmission path, in order to enhance the transmission capacity and the application flexibility of optical communication and sensor systems. The use of WDM technologies not only provides high speed optical communication links, but also offers advantages such as higher data rates, format transparency, and self-routing. Various types of available WDM devices include prisms, interference filters, and diffraction gratings. Currently modern optical networks use Arrayed Waveguide Grating (AWG) as optical wavelength (de)multiplexers [1] that use multiple waveguides to carry the optical signals. In this paper we report the use of a monolithic WDM device based on an a-Si:H/a-SiC:H multilayered semiconductor heterostructure. The device makes use of the fact that the

\footnotetext{
* Corresponding author. Tel.: +351218317287; fax: +351217114

E-mail address: plouro@deetc.isel.ipl.pt.
} 
optical absorption of the different wavelengths can be tuned by means of electrical bias changes or optical bias variations. This capability was obtained using adequate engineering design of the multiple layers thickness, absorption coefficient and dark conductivities [2,3]. The device described herein operates from 400 to $700 \mathrm{~nm}$ which makes it suitable for operation at visible wavelengths in optical communication applications. The device is a multilayered heterostructure based on a-Si:H and a-SiC:H. The configuration of the device includes two stacked p-i$\mathrm{n}$ structures between two electrical and transparent contacts (Fig. 1). Both front and back structures act as optical filters confining, respectively, the short and the long optical carriers, while the intermediate wavelengths are absorbed across both $[4,5]$. The device was operated within the visible range using as optical signals, to simulate the transmission optical channel, the modulated light (external regulation of frequency and intensity) supplied by a red, a green and a blue LED with wavelengths of $470 \mathrm{~nm}, 524 \mathrm{~nm}$ and $626 \mathrm{~nm}$, respectively. An electrical model of the WDM device is presented and supported by the solution of the respective circuit equations. Other possible applications of the device in optical communication systems are also proposed.

\section{Device configuration and multiplexing demultiplexing techniques}

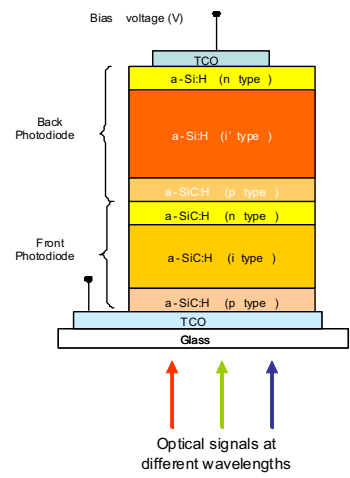

Fig. 1 WDM device configuration.

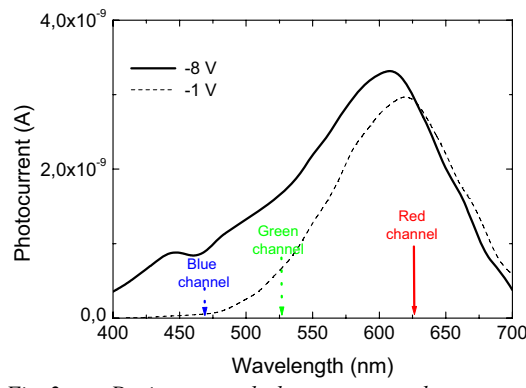

Fig. 2 Device spectral photocurrent under reverse and forward bias.
The devices were produced by PECVD and optimized for a proper fine tuning of a specific wavelength. The active device consists of a p-i'(a-SiC:H)-n / p-i(a-Si:H)-n heterostructure with low conductivity doped layers. The thicknesses and optical gap of the thin i'- $(200 \mathrm{~nm}$; $2.1 \mathrm{eV})$ and thick i- $(1000 \mathrm{~nm} ; 1.8 \mathrm{eV})$ layers are optimized for light absorption in the blue and red ranges, respectively. Transparent contacts have been deposited on front and back surfaces to allow the light to enter and leave from both sides (see Fig. 1).

To test the sensitivity of the device under different applied voltage and optical bias three modulated monochromatic lights channels: red (R: $626 \mathrm{~nm} ; 51 \mu \mathrm{W} / \mathrm{cm}^{2}$ ), green (G: $524 \mathrm{~nm} ; 73 \mu \mathrm{W} / \mathrm{cm}^{2}$ ) and blue (B: $\left.470 \mathrm{~nm} ; 115 \mu \mathrm{W} / \mathrm{cm}^{2}\right)$ and their polychromatic combinations (multiplexed signal) illuminated separately the device and the generated photocurrent was measured under positive and negative voltages $(+1 \mathrm{~V}<\mathrm{V}<-10 \mathrm{~V})$, with and without steady state green optical bias (G: $524 \mathrm{~nm} ; 73 \mu \mathrm{W} / \mathrm{cm}^{2}$ ). The light modulation frequency of

each channel was chosen to be multiple of the others to ensure a synchronous relation of $\mathrm{ON}-\mathrm{OFF}$ states along each cycle and the optical powers were adjusted to give different signal magnitudes at $8 \mathrm{~V}$.

Fig. 2 displays the spectral photocurrent under reverse and forward bias. Results show that in the long wavelengths range $(>600$ $\mathrm{nm})$ the spectral response is independent on the applied bias while in the short wavelength the collection strongly increases with the reverse bias.

Fig. 3 displays the single and multiplexed signals under negative $(-8 \mathrm{~V})$ and positive $(+1 \mathrm{~V})$ electrical bias. As expected from Fig. 2, the input red signal remains constant while the blue and the green ones decrease as the voltage changes from negative to positive.

The output multiplexed signal, obtained with the combination of the three optical sources, depends on both the applied voltage and on the ON-OFF state of each input optical channel Under negative bias, there are eight separate levels while under positive bias they were reduced to one half. The highest level appears when all the channels are $\mathrm{ON}$ and the lowest if they are OFF. Furthermore, the levels ascribed to the mixture of three or two input channels are higher than the ones due to the presence of only one (R, G, B). Optical nonlinearity was detected; the sum of the input channels $(\mathrm{R}+\mathrm{B}+\mathrm{G})$ is lower than the correspondent multiplexed signals (R\&G\&B). This optical amplification, mainly on the ON-ON states, suggests capacitive charging currents due to the time-varying nature of the incident 
lights. Under positive bias the levels are reduced to one half since and the blue component of the combined spectra falls into the dark level, the red remains constant and the green one decreases

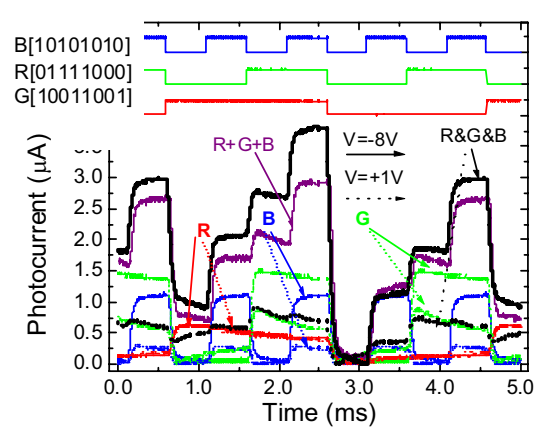

To recover the transmitted information ( 8 bit per wavelength channel) the multiplexed signal, during a complete cycle, was divided into eight time slots, each corresponding to one bit where the independent optical signals can be ON (1) or OFF (0).

Under positive bias, the device has no sensitivity to the blue channel (Fig. 1-2), so the red and green transmitted information are identified. The highest level corresponds to both channels ON (R\&G: $\mathrm{R}=1, \mathrm{G}=1)$, and the lowest to the OFF-OFF stage $(\mathrm{R}=0 ; \mathrm{G}=0)$. The two levels in-between are related with the presence of only one channel $\mathrm{ON}$, the red $(\mathrm{R}=1, \mathrm{G}=0)$ or the green $(\mathrm{R}=0, \mathrm{G}=1)$. To distinguish between these two situations and to decode the blue channel, the correspondent sub-levels, under reverse bias, have to be analyzed. The highest increase at $-8 \mathrm{~V}$ corresponds to the blue channel $\mathrm{ON}(\mathrm{B}=1)$, the lowest to the $\mathrm{ON}$ stage of the red channel $(\mathrm{R}=1)$ and the intermediate one to the $\mathrm{ON}$ stage of the green $(\mathrm{G}=1)$. Using this

Fig. $3 \quad$ Multiplexed signal (symbols) and experimental (solid lines) results under positive and negative dc bias. The input channels (symbols) are displayed at $-8 \mathrm{~V}$ and $+1 \mathrm{~V}$ simple key algorithm the independent red, green and blue bit sequences can be decoded as: R[01111000], G[10011001] and $\mathrm{B}[10101010]$, as shown on the top of Fig. 2, which are in agreement with the signals acquired for the independent channels.

\section{Two-transistor model and validation}

The silicon-carbon pi'npin device was considered as a monolithic double pin photodiode structure with two optical gate connections for light triggering. Operation is explained in terms of the compound connected phototransistor equivalent model displayed in Fig. 4 a.

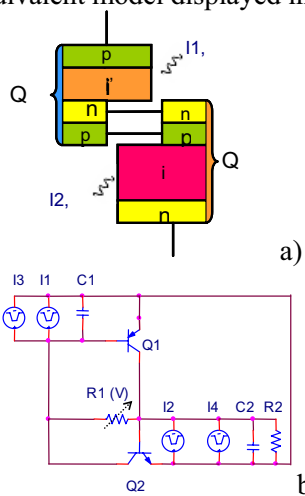

b)

The two-transistor model $\left(\mathrm{Q}_{1}-\mathrm{Q}_{2}\right)$ is obtained by bisecting the two middle layers in two separate halves that can be considered to constitute pnp $\left(\mathrm{Q}_{1}\right)$ and npn $\left(\mathrm{Q}_{2}\right)$ phototransistors separately. The first step was to determine the dc biasing condition for each stage, followed by $a c$ analysis using the proper equivalent circuits [8]. The circuit representation of the ac two-transistor model is displayed in Fig. 4b. When the pi'npin device is reverse-biased, the base-emitter junction of both transistors are inversely polarized and conceived as phototransistors, taking, so, advantage of the amplifier action of adjacent collector junctions which are polarized directly. This results in a current gain proportional to the ratio between both collector currents. Under positive bias the internal junction becomes reversebiased. If not triggered $\mathrm{ON}$ it is nonconducting, when turned $\mathrm{ON}$ by light it conducts like a photodiode, for one polarity of current. To trigger the device four square-wave current sources with different intensities are used; two , $\mathrm{I}_{1}$ and $\mathrm{I}_{2}$, with different frequencies to simulate the input blue and red channels and other two, $\mathrm{I}_{3}$ and $\mathrm{I}_{4}$, with the same frequency to simulate the green channel due to its asymmetrical absorption across both front and back phototr ansistorsThe charge stored in the space-charge layers is modeled by the capacitances $\mathrm{C}_{1}$ and $\mathrm{C}_{2}$, so, charging current across the reversed junctions $\left(i_{1,2}=C_{1,2} d v / d t\right)$ are considered. $R_{1}$ and $R_{2}$ model the dynamical resistances under different de bias conditions. The multiplexed signal was simulated by applying the Kirchhoff's laws for the simplified ac equivalent circuit and the four order Runge-Kutta method to solve the corresponding state equations. MATLAB was used as a programming environment

and the input parameters chosen in compliance with the experimental results (Fig. 3). The simulated transient currents (symbols) under negative and positive dc bias are displayed in Fig. 5. The same bit sequence of Fig. 3 was used. To validate the model the experimental multiplexed signals are also shown (solid lines).Good agreement between experimental and simulated data was observed. The eight expected levels, under reversed bias, and their reduction under forward bias (Fig. 3) are clearly seen. 


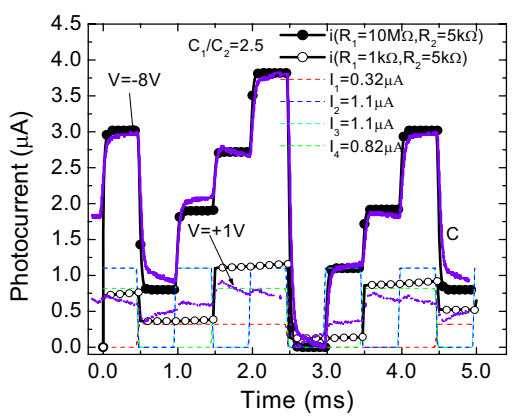

Fig. 5 Multiplexed simulated (symbols), current sources (dash lines) and experimental (solid lines): a) Positive (RI=10M $\Omega ;+1 \mathrm{~V})$ and negative dc bias. b) Negative dc bias $(R I=1 \mathrm{k} \Omega$; $-8 \mathrm{~V})$ with and without green irradiation.
The device behaves like a transmission system able to store and transport the minority carriers generated by the current pulses, through the capacitors $\mathrm{C}_{1}$ and $\mathrm{C}_{2}$. If not triggered $\mathrm{ON}$ (all the input channels OFF), either under positive or negative bias, the device is nonconducting (low level). Under negative bias (low $\mathrm{R}_{1}$ ) and if no optical bias is applied, the expected eight levels are detected, each one corresponding to the presence of three, two, one or no color channel $\mathrm{ON}$. If $\mathrm{I}_{1}$ or $\mathrm{I}_{2}$ are $\mathrm{ON}, \mathrm{C}_{2}$ and $\mathrm{C}_{1}$ are rapidly charged in inverse polarity. The current source keeps filling the capacitors during the pulse and the transferred charge between them reaches the output terminal as a capacitive charging current. An optical amplification is observed due to the amplifier action of adjacent collector junctions which are always polarized directly. With all the input channels $\mathrm{ON}$, the packets of charge stored at $\mathrm{C}_{1}\left(\mathrm{I}_{1}, \mathrm{I}_{3}\right)$ are sequentially transferred to $\mathrm{C}_{2}$ and together with the minority carriers generated at the base of $\mathrm{Q}_{2}\left(\mathrm{I}_{2}, \mathrm{I}_{4}\right)$ flow across the circuit. Under forward bias (high $\mathrm{R}_{1}$ ) the device remains in its non conducting state unless a light pulse $\left(\mathrm{I}_{2}\right.$ or $\left.\mathrm{I}_{2}+\mathrm{I}_{4}\right)$ is applied to the

base of $\mathrm{Q}_{2}$. This pulse causes $\mathrm{Q}_{2}$ to conduct because the reversed biased n-p internal junction behaves like a capacitor inducing a charging current $\left(\mathrm{I}_{2}+\mathrm{I}_{4}\right)$ across both collector junctions. The collector of the conducting transistor pulls low, moving the $\mathrm{Q}_{1}$ base toward its collector voltage, which causes $\mathrm{Q}_{1}$ to conduct. The collector of the conducting $\mathrm{Q}_{1}$ pulls high, moving the $\mathrm{Q}_{2}$ base in the direction of its collector. This positive feedback (regeneration) reinforces the $\mathrm{Q}_{2}$ already conducting state and a current $\mathrm{I}_{2}+\mathrm{I}_{4}$ will flow on the external circuit.

Results show that the two-transistor model explains the difference between the conduction mechanisms, under positive and negative bias, helping to understand the signal decoding algorithm. Under positive bias the red and green channels are immediately decoded since the collected current is due exclusively to the generated carriers at the back diode. Under negative bias, depending on the ratio between $C_{1}$ and $C_{2}$, different charging currents across the reversed junctions have to be considered. The balance between them depends on the presence of three, two or one channel ON. So, by comparing the different sublevels signals under positive and negative bias it is possible to recover the blue, the green and the red input channels.

\section{Conclusions}

A double pi'n/pin a-SiC:H heterostructure with two optical gate connections for light triggering in different spectral regions was presented. Multiple monochromatic pulsed communication channels, in the visible range, were transmitted together, each one with a specific bit sequence. The combined optical signal was analyzed by reading out, under positive and negative voltages and optical green bias, the generated photocurrent across the device. Results show that the output multiplexed signal has a strong nonlinear dependence on the light absorption profile, i.e. on the incident light wavelength, bit rate, intensity and optical bias due to the self biasing of the junctions under unbalanced light generation profiles. By switching between positive and negative voltages the input channels can be recovered or removed.

\section{References}

[1] M.Bas, Fiber Optics Handbook, Fiber, Dev.and Syst. for Opt. Comm., Chap, 13, Mc Graw-Hill, 2002.

[2] P. Louro, M. Vieira, Yu. Vygranenko, A. Fantoni, M. Fernandes, G. Lavareda, N. Carvalho Mat. Res. Soc. Symp. Proc., 989 (2007) A12.04.

[3] M. Vieira, M. Fernandes, P. Louro, A. Fantoni, Y. Vygranenko, G. Lavareda, C. Nunes de Carvalho, Mat. Res. Soc. Symp. Proc., Vol. 862 (2005) A13.4.

[4] P. Louro, M. Vieira, M.A. Vieira, M. Fernandes, A. Fantoni, C. Francisco, M. Barata, Physica E: Low-dimensional Systems and Nanostructures, 41 (2009) 1082-1085.

[5] P. Louro, M. Vieira, M. Fernandes, J. Costa, M. A. Vieira, J. Caeiro, N. Neves, M. Barata, Phys. Status Solidi C 7, No. 3-4, $1188-1191$ (2010). 